\title{
Erratum to: CXCL13-CXCR5 co-expression regulates epithelial to mesenchymal transition of breast cancer cells during lymph node metastasis
}

\author{
Subir Biswas ${ }^{1}$ - Suman Sengupta ${ }^{1}$ - Sougata Roy Chowdhury ${ }^{2} \cdot$ Samir Jana $^{1}$ • \\ Gunjan Mandal ${ }^{1}$ - Palash Kumar Mandal ${ }^{3}$ - Nipun Saha ${ }^{4}$. Vivek Malhotra ${ }^{4}$. \\ Arnab Gupta ${ }^{4}$ Dmitry V. Kuprash ${ }^{5}$ - Arindam Bhattacharyya ${ }^{1}$
}

Published online: 17 February 2016

(C) Springer Science+Business Media New York 2016

\section{Erratum to: Breast Cancer Res Treat (2014) 143:265-276 \\ DOI 10.1007/s10549-013-2811-8}

In the original publication of the article, the Fig. 6C-i and ii were published incorrectly. The correct immunoblot images of Vimentin (for ROE+LT MDA-MB-231) and N-Cadherin (for ROE+LT T-47D) against Src and
PI3Kp110a inhibitors SU6656 and PI-103 are now given in Fig. 6C-i and ii with reanalyzed densitometry. These corrections do not change the interpretation of the results or the conclusions of this work.

The online version of the original article can be found under doi:10.1007/s10549-013-2811-8.

Arindam Bhattacharyya

arindam19@yahoo.com

1 Immunology Laboratory, Department of Zoology, University of Calcutta, 35, Ballygunge Circular Road, Kolkata 700019, West Bengal, India

2 Materials Science Centre, Indian Institute of Technology Kharagpur, Kharagpur, India

3 Department of Pathology, North Bengal Medical College, Darjeeling, India

4 Department of Surgical Oncology, Saroj Gupta Cancer Centre and Research Institute, Kolkata, India

5 Laboratory of Immunoregulation, Engelhardt Institute of Molecular Biology, Russian Academy of Sciences, Moscow, Russia 

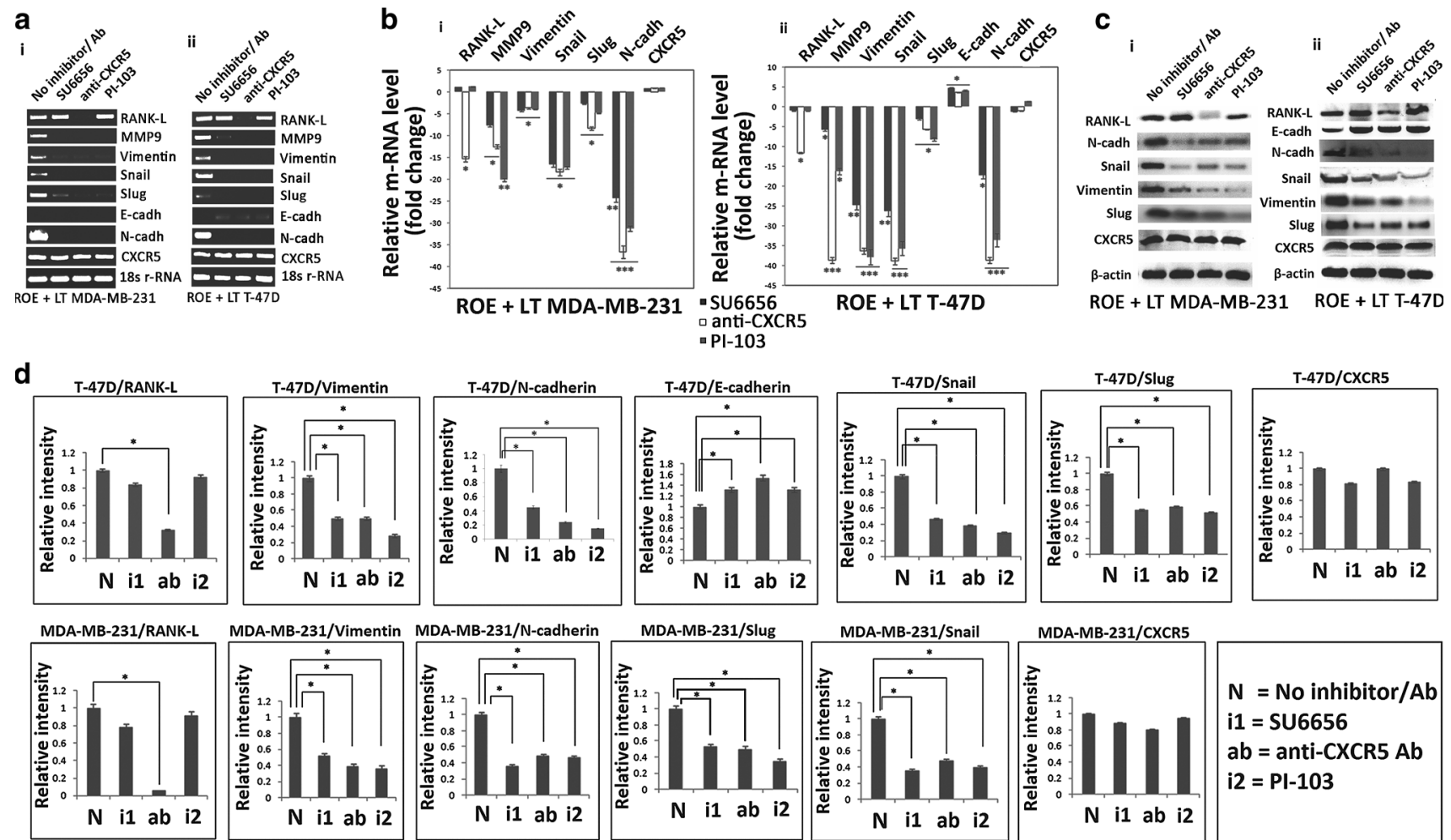

$\mathbf{N}=$ No inhibitor $/ \mathrm{Ab}$ i1 $=$ SU6656 $\mathrm{ab}=$ anti-CXCR5 Ab i2 $=$ PI-103

Fig. 6 Involvement of RANKL, Src, PI3Kp110 $\alpha$ in CXCL13induced EMT and MMP9 expression. Prior to CXCL13 treatment, ROE + LT cells were treated with inhibitors of Src and PI3Kp110 $\alpha$, i.e., SU6656 and PI-103, respectively, or with anti-CXCR5 monoclonal antibody. a mRNA levels of Vimentin, Slug, Snail, E-cadh, $\mathrm{N}$-cadh, CXCR5, RANKL, and MMP9 were assessed by conventional RT-PCR, followed by agarose gel electrophoresis. 18s r-RNA was used as internal control. b Quantitative real-time RT-PCR was performed for these mRNAs. Fold changes are represented as relative values $\left(2^{-\Delta \Delta \mathrm{Ct}}\right)$ for SU6656, anti-CXCR5-antibody, PI-103-treated ROE + LT cells normalized with internal control and quantified. Fold changes in MDA-MB-231 were as follows: Vimentin decreased 4.4, 3.7, 4.0-fold, respectively; N-cadh decreased 22.5, 31.8, 36.3fold, respectively; Snail decreased 16.1, 17.2, 16.7-fold, respectively; Slug decreased 2.2, 8.33, 3.9-fold, respectively; and MMP9 decreased 7.7, 12.5, 20.0-fold, respectively. For T-47D cells, fold changes were as follows: Vimentin decreased 24.9, 37.3, 38.5-fold, respectively; $\mathrm{N}$-cadh decreased 17.3, 38.6, 34.2-fold, respectively; Snail decreased
26.0, 38.5, 35.1-fold, respectively; Slug decreased 3.1, 5.7, 8.2-fold, respectively; and MMP9 was decreased 5.9, 37.3, 16.5-fold, respectively. E-cadh expression increased significantly in all the treatment conditions for T-47D cells. RANKL expression, however, decreased significantly only upon anti-CXCR5 treatment. No treatment had any effect on CXCR5 mRNA level. c Expressions of Vimentin, Slug, Snail, N-cadh, E-cadh, RANKL, and CXCR5 were evaluated by immunoblotting. $\beta$-actin was used as loading control. Vimentin, Slug, Snail, and N-cadh expressions were decreased in SU6656, PI-103, anti-CXCR5-antibody treated ROE + LT cells. Inhibitor/antiCXCR5-antibody treated ROE + LT T-47D cells have decreased E-cadh expression. RANKL expression was only found to be downregulated in anti-CXCR5-antibody treated ROE + LT cells. d WB band densitometries shown in bar graphs. Results are representative of three independent experiments performed in triplicate and are represented as mean $\pm \mathrm{SD}$. One-way ANOVA (Bonferroni correction) was performed, where significance level stands for $* p \leq 0.05$, $* * p \leq 0.01, * * * p \leq 0.001$ 